\title{
Vibration Analysis of Cylindrical Sandwich Aluminum Shell with Viscoelastic Damping Treatment
}

\author{
Tai-Hong Cheng, Zhen-Zhe Li, and Yun-De Shen \\ College of Mechanical and Electrical Engineering, Wenzhou University, Zhejiang Province 325035, China \\ Correspondence should be addressed to Yun-De Shen; shenyunde63@163.com
}

Received 11 July 2013; Accepted 6 September 2013

Academic Editor: Yan Yang

Copyright ( 2013 Tai-Hong Cheng et al. This is an open access article distributed under the Creative Commons Attribution License, which permits unrestricted use, distribution, and reproduction in any medium, provided the original work is properly cited.

This paper has applied the constrained viscoelastic layer damping treatments to a cylindrical aluminum shell using layerwise displacement theory. The transverse shear, the normal strains, and the curved geometry are exactly taken into account in the present layerwise shell model, which can depict the zig-zag in-plane and out-of-plane displacements. The damped natural frequencies, modal loss factors, and frequency response functions of cylindrical viscoelastic aluminum shells are compared with those of the base thick aluminum panel without a viscoelastic layer. The thickness and damping ratio of the viscoelastic damping layer, the curvature of proposed cylindrical aluminum structure, and placement of damping layer of the aluminum panel were investigated using frequency response function. The presented results show that the sandwiched viscoelastic damping layer can effectively suppress vibration of cylindrical aluminum structure.

\section{Introduction}

The cylindrical curved structure has been employed in many engineering applications, such as aircrafts, automobiles, ships, and other industrial machines. Which structures are usually vibrated with different level due to external regular or random loads. And the structural failures can originate from the vibration by severe dynamic load and structural resonances. So the study of vibration and dynamic characteristics of cylindrical structure is an important work in engineering field.

Numerical researches have been studied for vibration of the shells with different theories. Studies on the vibrations characteristics of the cylindrical shells have been carried out extensively using numerical methods. Love [1] modified the Kirchhoff hypothesis for plates and established the assumptions used in the so-called classic theory of thin shells. Arnold and Warburton, Chung, Bhimaraddi, Soldatos, and Hadjigeorgiou analyzed vibration of a cylindrical structure based on Love hypothesis [2-5]. Constrained layer damping is a mechanical engineering technique for suppression of vibration. Typically a viscoelastic or another damping material is sandwiched between two sheets of stiff materials that lack sufficient damping by themselves. Ross et al. presented a general analysis of the viscoelastic structures [6]. They described the damping mechanism of the viscoelastic material as in-plane and transverse shear deformations, RKU assumptions. Mead and Markus developed sixth-order equations of motion for the transverse displacement of the damped sandwich beams with arbitrary boundary conditions [7]. Blasingame and DiTaranto added the extensional stiffness of the dissipative core layer to Kerwin's formulation of the laminated sandwich plate [8]. Siu and Bert investigated the material damping of laminated anisotropic rectangular plates, considering the thickness-shear flexibility and rotary and coupling inertia [9]. Chen and Huang presented a study on optimal placement of PCLD treatment for vibration suppression of the plates [10]. In their optimization, the structural damping plays the main performance index and the frequencies' shift and constraint layer damping thickness act as penalty functions. Zheng et al. have adopted genetic algorithm based penalty function method that is employed to find the optimal layout of rectangular passive constrained layer damping patches aiming to minimize the structural volume displacement 


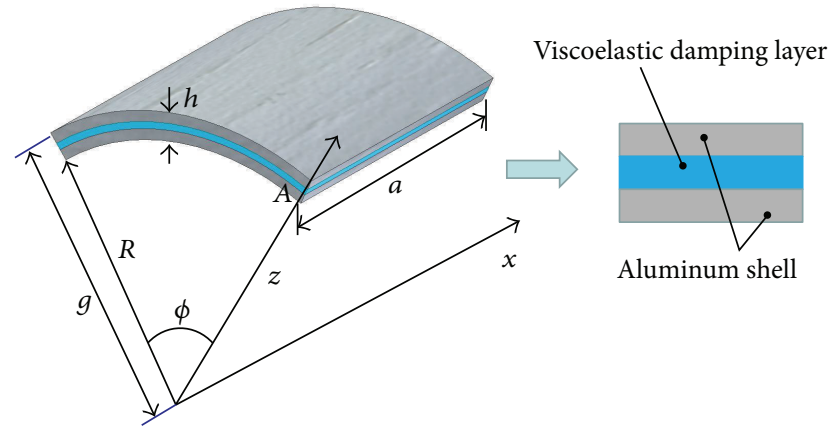

FIGURE 1: Geometry of cylindrical aluminum shell with viscoelastic damping layer.

of constraint layer damping treated cylindrical shell [11]. An optimization solution of rectangular constraint layer damping patches locations and dimensions is obtained under the constraint of total amount of constraint layer damping in terms of percentage added weight to the base structure. Ro and Baz presented optimal damping studies with an active constrained viscoelastic damping treatment distributed over regions of high strain energy on a plate [12]. Masti and Sainsbury have investigated the effectiveness of using a strain energy-based partial coating approach for vibration attenuation of the cylindrical shells [13]. The isogeometric analysis with nonuniform rational B-spline based on the classical plate theory is developed for free vibration analyses of functionally graded material thin plates [14].

In this study, a viscoelastic constrained layer damping treatment was employed to minimize vibration of a cylindrical aluminum panel using layerwise finite element theory, the base structure, and the constraining layer. The transverse shear, the normal strains, and the curved geometry are exactly taken into account in the present layerwise shell model, which can depict the zig-zag in-plane and out-ofplane displacements. The frequency response functions, the mode shapes, and the modal loss factor of a cylindrical sandwich aluminum panel were investigated with viscoelastic damping treatment.

\section{Description of Finite Element Modeling}

Figure 1(a) shows the geometry of a cylindrical aluminum shell with a viscoelastic damping layer. In this study, the layerwise shell theory is proposed to accurately predict the vibration and damping characteristics of the cylindrical aluminum panels with the viscoelastic damping layers. Based on the full layerwise shell theory, the displacement fields $(u, v$, and $w$ ) on the cylindrical coordinate system can be expressed by introducing the piecewise interpolation function along the thickness direction and finite element shape functions
Interpolation functions $\quad u(x, \phi, z, t)=\sum_{J=1}^{N_{i}} U^{J}(x, \phi, t) \Phi^{J}(z)$

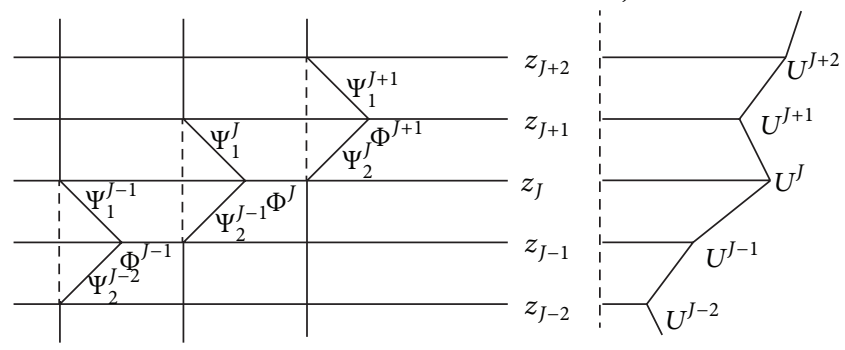

FIGURE 2: In-plane displacement based on the layerwise theory.

$$
\begin{gathered}
u(x, \phi, z, t)=\sum_{J=1}^{N i} U^{J}(x, \phi, t) \Phi^{J}(z), \\
v(x, \phi, z, t)=\sum_{J=1}^{N i} V^{J}(x, \phi, t) \Phi^{J}(z), \\
w(x, \phi, z, t)=\sum_{J=1}^{N i} W^{J}(x, \phi, t) \Phi^{J}(z),
\end{gathered}
$$

where the linear interpolation function $\Phi^{J}(z)$ along the thickness direction can be expressed by the following form:

$$
\Phi^{J}(z)= \begin{cases}0 & \text { for } z<z_{J-1}, \\ \Psi_{2}^{J-1}(z)=\frac{z-z_{J-1}}{z_{J}-z_{J-1}}, & \text { for } z_{J-1}<z<z_{J} \\ \Psi_{1}^{J}(z)=-\frac{z-z_{J+1}}{z_{J+1}-z_{J}}, & \text { for } z_{J}<z<z_{J+1} \\ 0, & \text { for } z_{J+1}<z .\end{cases}
$$

Based on the sublaminate layerwise shell theory, an inplane displacement can be described with a zig-zag deformation along the thickness direction as shown in Figure 2 [15]. The relationship between strains and displacement fields can be expressed as follows:

$$
\begin{gathered}
\varepsilon_{x x}=\frac{\partial u}{\partial x}=\sum_{J=1}^{N i} \frac{\partial U^{J}}{\partial x} \Phi^{J} \\
\varepsilon_{\phi \phi}=\frac{1}{g_{\phi}}\left(\frac{\partial v}{\partial \phi}+w\right)=\frac{1}{g_{\phi}} \sum_{J=1}^{N i}\left(\frac{\partial V^{J}}{\partial \phi}+W^{J}\right) \Phi^{J} \\
\varepsilon_{z z}=\frac{\partial w}{\partial z}=\sum_{J=1}^{N i} W^{J} \frac{d \Phi^{J}}{d z}
\end{gathered}
$$




$$
\begin{aligned}
& \varepsilon_{\phi z}=\frac{1}{g_{\phi}}\left(\frac{\partial w}{\partial \phi}-v\right)+\frac{\partial v}{\partial z} \\
&=\frac{1}{g_{\phi}} \sum_{J=1}^{N i}\left(\frac{\partial W^{J}}{\partial \phi}-V^{J}\right) \Phi^{J}+\sum_{J=1}^{N i} V^{J} \frac{d \Phi^{J}}{d z} \\
& \varepsilon_{x z}=\frac{\partial w}{\partial x}+\frac{\partial u}{\partial z}=\sum_{J=1}^{N i}\left(\frac{\partial W^{J}}{\partial x} \Phi^{J}+U^{J} \frac{d \Phi^{J}}{d z}\right) \\
& \varepsilon_{x \phi}=\frac{\partial v}{\partial x}+\frac{1}{g_{\phi}} \frac{\partial u}{\partial \phi}=\sum_{J=1}^{N i}\left(\frac{\partial V^{J}}{\partial x}+\frac{1}{g_{\phi}} \frac{\partial U^{J}}{\partial \phi}\right) \Phi^{J}
\end{aligned}
$$

$g_{\phi}$ is defined as a function of $z$ in the following form:

$$
g_{\phi}=R\left(1+\frac{z}{R}\right)=R+z
$$

\section{Governing Equations of Cylindrical Panel}

To derive the governing equation of motion for the cylindrical panels with the viscoelastic layers, Hamilton's variational principle was applied in the following form:

$$
\underbrace{\int_{V} \rho \ddot{u}_{i} \delta u_{i} d V}_{\delta T(\text { Kinetic Energy })}+\underbrace{\int_{V} \sigma_{i j} \delta \varepsilon_{i j} d V}_{\delta V(\text { Strain Energy })}=\underbrace{\int_{V} f_{i} \delta u_{i} d V+\int_{S} \tau_{i} \delta u_{i} d S}_{\delta W \text { (External Works) }},
$$

where $\rho, f_{i}$, and $\tau_{i}$ are the density, body force, and surface force, respectively. The displacement filed of $J$ th interface was defined using shape function as follows:

$$
\begin{aligned}
U^{J}(x, \phi, t) & =\sum_{I=1}^{\mathrm{NPE}} U_{I}^{J}(t) \bar{\psi}_{I}(x, \phi), \\
V^{J}(x, \phi, t) & =\sum_{I=1}^{\mathrm{NPE}} V_{I}^{J}(t) \bar{\psi}_{I}(x, \phi), \\
W^{J}(x, \phi, t) & =\sum_{I=1}^{\mathrm{NPE}} W_{I}^{J}(t) \bar{\psi}_{I}(x, \phi),
\end{aligned}
$$

where NPE is node per element and $\psi_{I}$ is linear interpolation function and by substituting (1a), (1b), (1c), (3a), (3b), (3c), $(3 \mathrm{~d}),(3 \mathrm{e})$, and (3f) into (5), we can obtain the following equations of the finite element:

$$
\mathbf{M}_{e} \ddot{\mathbf{u}}_{e}+\mathbf{K}_{e} \mathbf{u}_{e}=\mathbf{F}_{e} .
$$

Consequently, we can obtain the global finite element equations of motion through the assembly process, resulting in the following complex form:

$$
\mathbf{M U}+\mathbf{K U}=\mathbf{F} .
$$

\section{Dynamic Equation of Cylindrical Panel with Viscoelastic Damping Layer}

Here, normal and shear moduli can be expressed in the form of complex numbers as follows:

$$
\begin{gathered}
\bar{E}_{I I}=E_{I I}\left(1+i \eta_{I I}\right) \quad \text { for } I=1,2,3 \\
\bar{G}_{12}=G_{12}\left(1+i \eta_{12}\right), \\
\bar{G}_{23}=G_{23}\left(1+i \eta_{23}\right), \quad \bar{G}_{13}=G_{13}\left(1+i \eta_{13}\right) .
\end{gathered}
$$

By considering the complex modulus of (9a) and (9b), the eigensystem matrices for undamped free vibration can be written in the following form:

$$
\mathbf{M} \ddot{\mathbf{U}}+\left(\mathbf{K}(\boldsymbol{\omega})+\mathbf{i} \mathbf{K}_{\mathbf{D}}(\boldsymbol{\omega})\right) U=\mathbf{F}(\boldsymbol{\omega}) .
$$

The modal approach using the eigensolutions of undamped free vibration is applied to reduce the order of system matrices as follows:

$$
\mathbf{M}^{*} \ddot{\mathbf{U}}^{*}+\left(\mathbf{K}^{*}(\boldsymbol{\omega})+\mathbf{i} \mathbf{K}_{D}^{*}(\boldsymbol{\omega})\right) U^{*}=\mathbf{F}^{*}(\boldsymbol{\omega}),
$$

where the reduced system matrices and vectors are given as

$$
\begin{gathered}
\mathbf{M}^{*}=\Psi^{T} \mathbf{M} \Psi, \\
\mathbf{K}^{*}=\Psi^{T} \mathbf{K} \Psi, \quad \mathbf{K}_{D}^{*}=\Psi^{T} \mathbf{K}_{D} \Psi, \\
\mathbf{F}^{*}=\Psi^{T} \mathbf{F}, \\
\mathbf{U}=\Psi \mathbf{U}^{*} .
\end{gathered}
$$

From (11), natural frequencies and modal loss factors can be defined as follows:

$$
\omega_{n}^{2}=\operatorname{Real}\left[\lambda_{n}^{*}\right], \quad \eta_{n}=\frac{\operatorname{Imag}\left[\lambda_{n}^{*}\right]}{\operatorname{Real}\left[\lambda_{n}^{*}\right]} .
$$

The frequency response function is used to investigate the steady state dynamic characteristics of the linear system subject to harmonic excitation. Due to a harmonic excitation at a certain point, the reduced force vector with respect to modal coordinates is given as follows:

$$
\mathbf{F}^{*}=\Psi^{T} \mathbf{F}=F_{0} \Psi^{T} \mathbf{F}_{\text {input }} e^{i \omega t}=F_{0} \mathbf{F}_{\text {input }}^{*} e^{i \omega t} .
$$

Finally, the frequency response function obtained from the modal approach is given in the following form:

$$
\begin{aligned}
\mathbf{H} & =\frac{\mathbf{U}}{F_{o}}=\frac{\Psi \mathbf{U}^{*}}{F_{o}} \\
& =\Psi\left(\mathbf{K}^{*}(\omega, T)+i \mathbf{K}_{D}^{*}(\omega, T)-\omega^{2} \mathbf{M}^{*}\right)^{-1} \mathbf{F}_{\text {Input }}^{*},
\end{aligned}
$$

$$
\text { Magnitude }=|\mathbf{H}| \text {, }
$$

$$
\text { Phase, } \varphi=\arctan \left(-\frac{\operatorname{Imag}(\mathbf{H})}{\operatorname{Real}(\mathbf{H})}\right) \text {. }
$$


No damping layer (NDL)

Sandwich single damping layer (SSDL)

Sandwich double damping layer (SDDL)

Aluminum

Viscoelastic damping layer

FIGURE 3: Three cases discussed in this study.

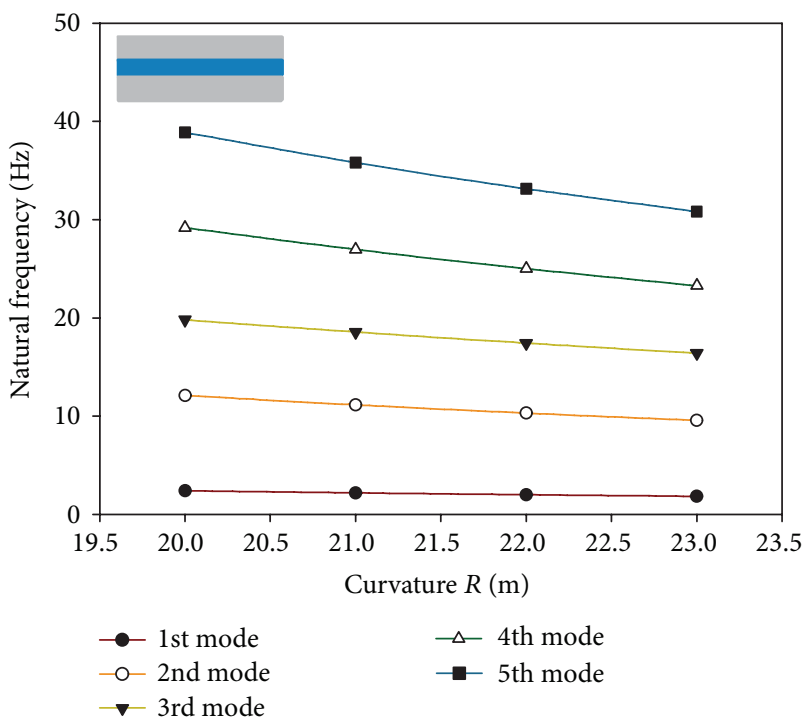

FIGURE 4: Variation of natural frequencies of cylindrical aluminum panel in case of SSDL with variation of curvature $R(a=0.2 \mathrm{~m}, b=$ $0.1 \mathrm{~m}, t_{1}=5 \mathrm{~mm}, t_{2}=2 \mathrm{~mm}, t_{3}=5 \mathrm{~mm}$, and $\left.\eta=0.1\right)$.

TABLE 1: Material properties of aluminum and viscoelastic material.

\begin{tabular}{lccc}
\hline & Elastic modulus & Poisons ratio & Density \\
\hline Aluminum & $69 \mathrm{GPa}$ & 0.3 & $2800 \mathrm{~kg} / \mathrm{m}^{3}$ \\
Viscoelastic material & $2.1 \mathrm{Mpa}$ & 0.499 & $970 \mathrm{~kg} / \mathrm{m}^{3}$ \\
\hline
\end{tabular}

\section{Results and Discussion}

For finite element analysis of the cylindrical aluminum panel, the nine-node $2 \times 4$ meshes are used for the cylindrical panel and the material properties are given as in Table 1 .

Figure 3 shows the three discussed cases in this study, which are no damping layer (NDL), sandwich single damping layer (SSDL), and sandwich double damping layer (SDDL). Figures 4 and 5 show the variation of the natural frequencies and the modal loss factor of cylindrical aluminum panel in case of SSDL with variation of the curvature $R$. The

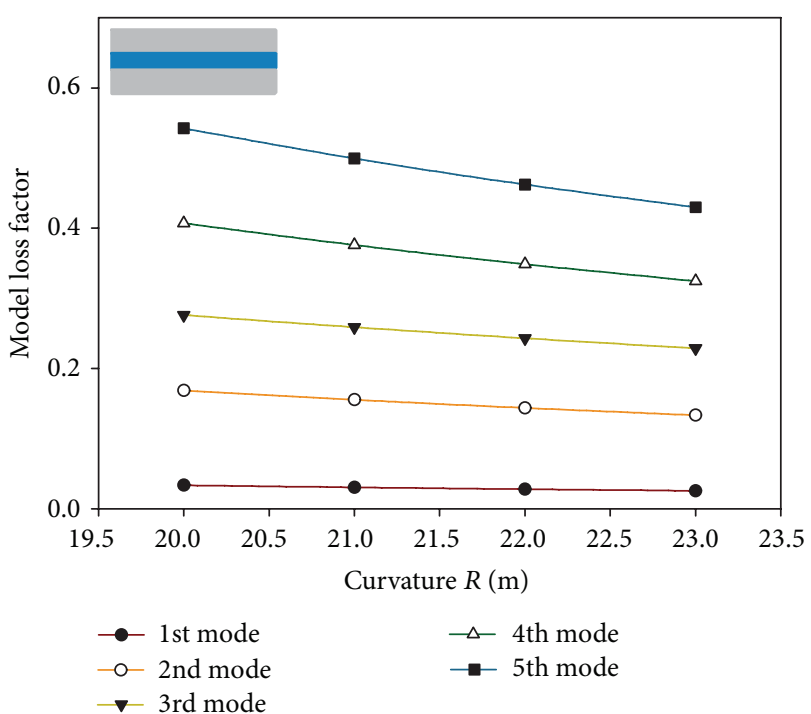

FIGURE 5: Variation of modal loss factor of cylindrical aluminum panel in case of SSDL with variation of curvature $R(a=0.2 \mathrm{~m}, b=$ $0.1 \mathrm{~m}, t_{1}=5 \mathrm{~mm}, t_{2}=2 \mathrm{~mm}, t_{3}=5 \mathrm{~mm}$, and $\left.\eta=0.1\right)$.

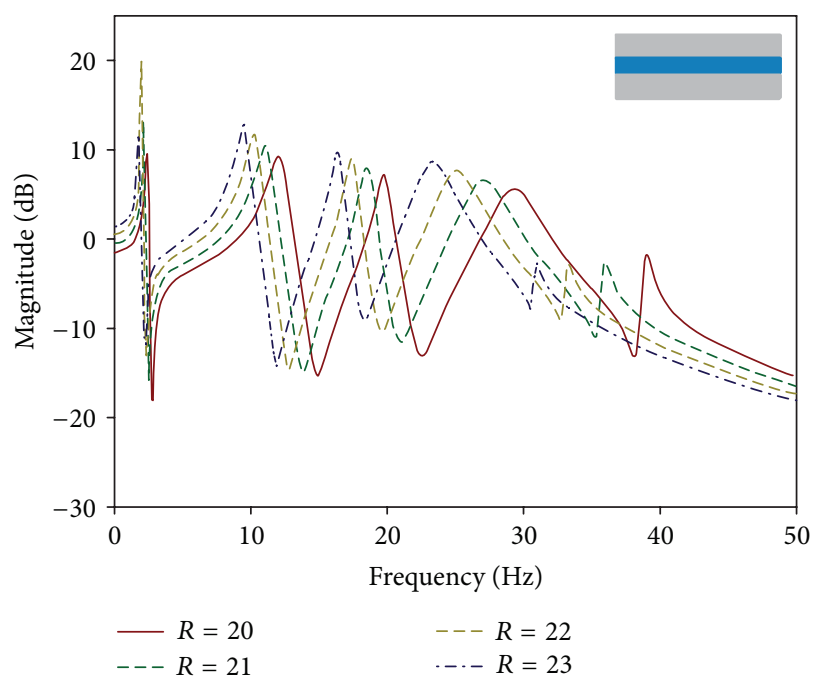

FIGURE 6: Frequency response of cylindrical aluminum panel in case of SSDL with variety of curvatures $R\left(a=0.2 \mathrm{~m}, b=0.1 \mathrm{~m}, t_{1}=\right.$ $5 \mathrm{~mm}, t_{2}=2 \mathrm{~mm}, t_{3}=5 \mathrm{~mm}$, and $\left.\eta=0.1\right)$.

natural frequencies and the modal loss factor are decreased with increasing $R$ except the first mode. Figure 6 shows the frequency response of the cylindrical aluminum panel in case of SSDL with variety of the curvatures $R$. As the radius of the cylindrical geometry was increased, the natural frequencies decreased except that the first mode and the magnitude of the second, the third, the fourth, and the fifth modes are increased due to the dominance of the transverse shear and the curvature effect.

Figure 7 shows the frequency response of the cylindrical aluminum panel in case of SSDL with variety of the central angles $\phi$. As the central angle of the cylindrical geometry was increased, the natural frequencies decreased except 


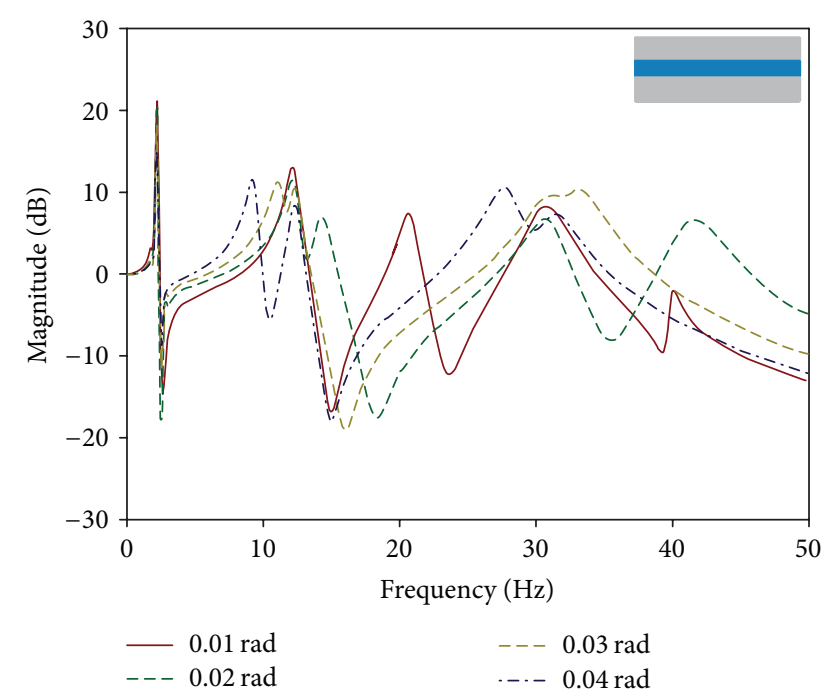

FIGURE 7: Frequency response of cylindrical aluminum panel in case of SSDL with variety of central angles $\phi(a=0.2 \mathrm{~m}, R=20 \mathrm{~m}$, $t_{1}=5 \mathrm{~mm}, t_{2}=2 \mathrm{~mm}, t_{3}=5 \mathrm{~mm}$, and $\left.\eta=0.1\right)$.

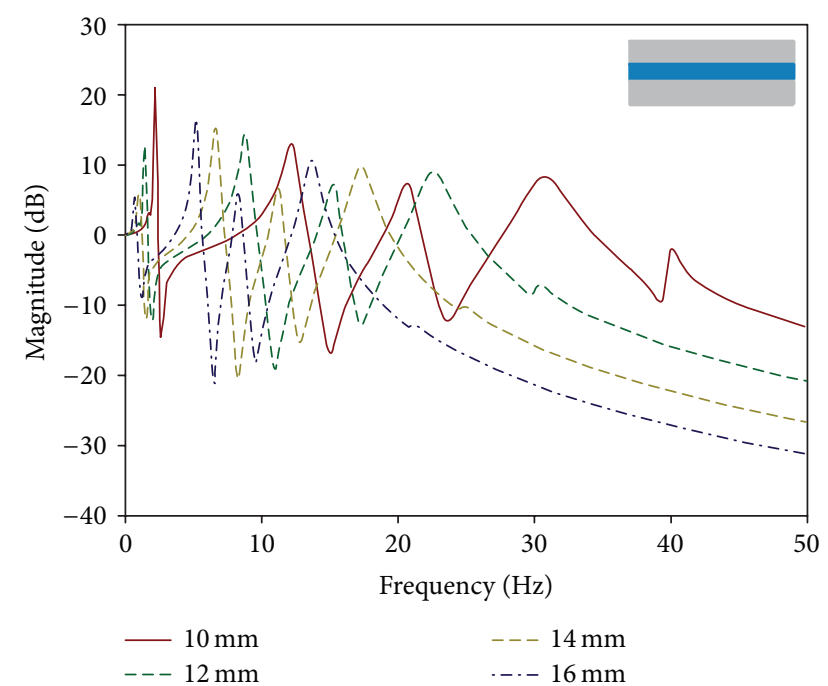

FIGURE 8: Frequency response of cylindrical aluminum panel in case of SSDL with variety of lengths $a\left(\phi=0.01 \mathrm{rad}, R=20 \mathrm{~m}, t_{1}=\right.$ $5 \mathrm{~mm}, t_{2}=2 \mathrm{~mm}, t_{3}=5 \mathrm{~mm}$, and $\left.\eta=0.1\right)$.

that the first mode and especially the second mode are more dominant with the central angle $\phi$. Figure 8 shows the frequency response of the cylindrical aluminum panel in case of SSDL with variety of the lengths $a$. With increasing the length value $a$ the natural frequency has a decreasing trend in the frequency response function. The damping factor is an important parameter in the viscoelastic material. Figure 9 shows the frequency response of the cylindrical aluminum panel in case of SSDL with variety of the damping factors $\eta$ of the viscoelastic damping material. As the damping factor increases, the magnitude of the first five modes decreased, because of good damping performance of the viscoelastic damping layer. Figure 10 shows the frequency response of

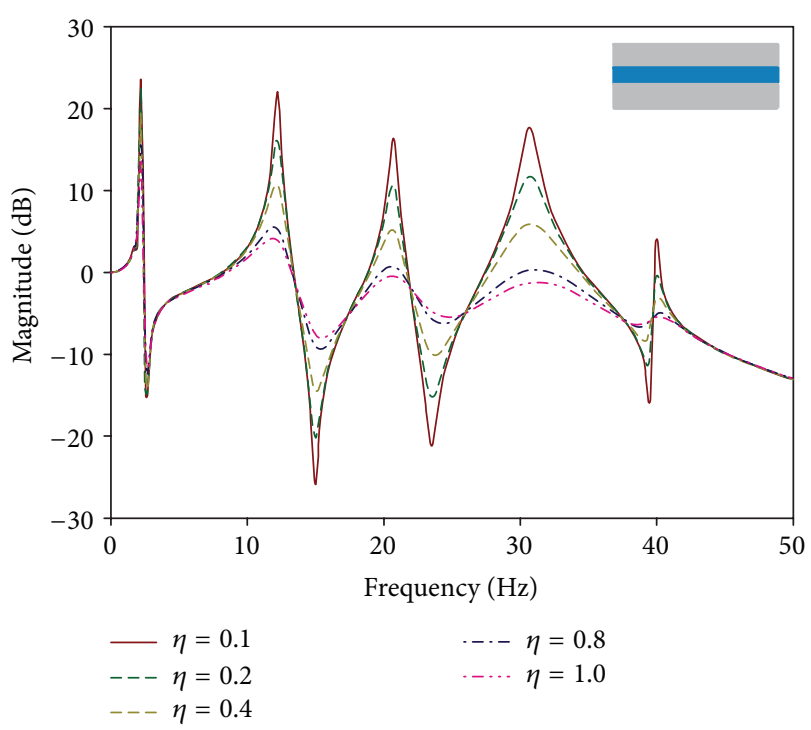

FIGURE 9: Frequency response of cylindrical aluminum panel in case of SSDL with variety of damping factors $\eta$ of viscoelastic material $\left(a=0.2 \mathrm{~m}, \phi=0.01 \mathrm{rad}, R=20 \mathrm{~m}, t_{1}=5 \mathrm{~mm}, t_{2}=2 \mathrm{~mm}\right.$, and $\left.t_{3}=5 \mathrm{~mm}\right)$

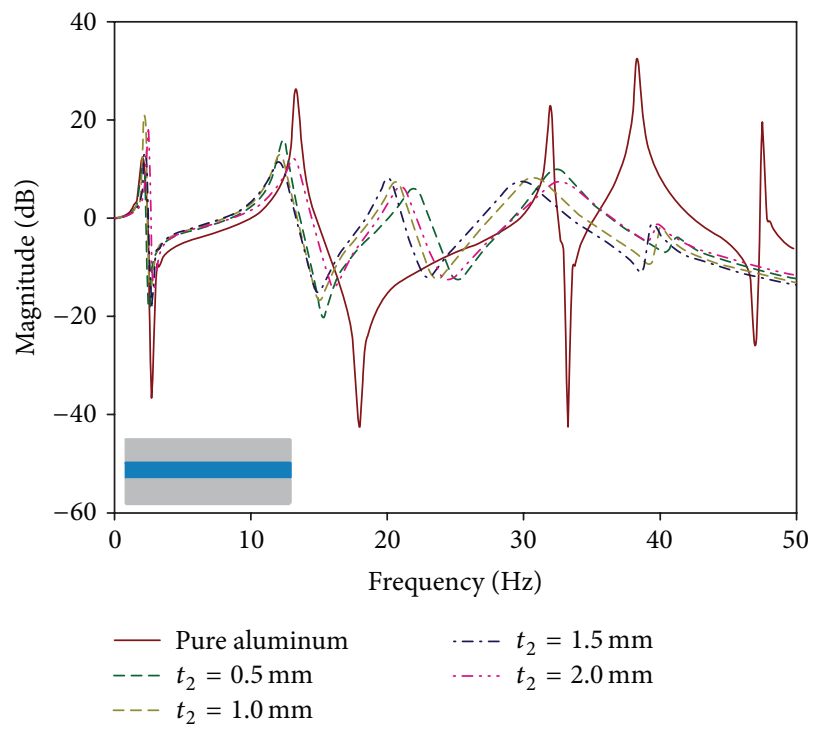

FIGURE 10: Frequency response of cylindrical aluminum panel in case of SSDL with variety of thicknesses of damping layer $t_{2}(a=$ $0.2 \mathrm{~m}, \phi=0.01 \mathrm{rad}, R=20 \mathrm{~m}, t a=10 \mathrm{~mm}, t_{1}=5 \mathrm{~mm}, t_{3}=5 \mathrm{~mm}$, and $\eta=0.1$ ).

the cylindrical aluminum panel in case of SSDL with variety of the thicknesses of the damping layer $t 2$. The magnitude of SSDL has good damping effect at the second, the third, the fourth, and the fifth modes, with shifting of the natural frequency. Figure 11 shows the frequency response function of the cylindrical aluminum panel in case of SSDL with 


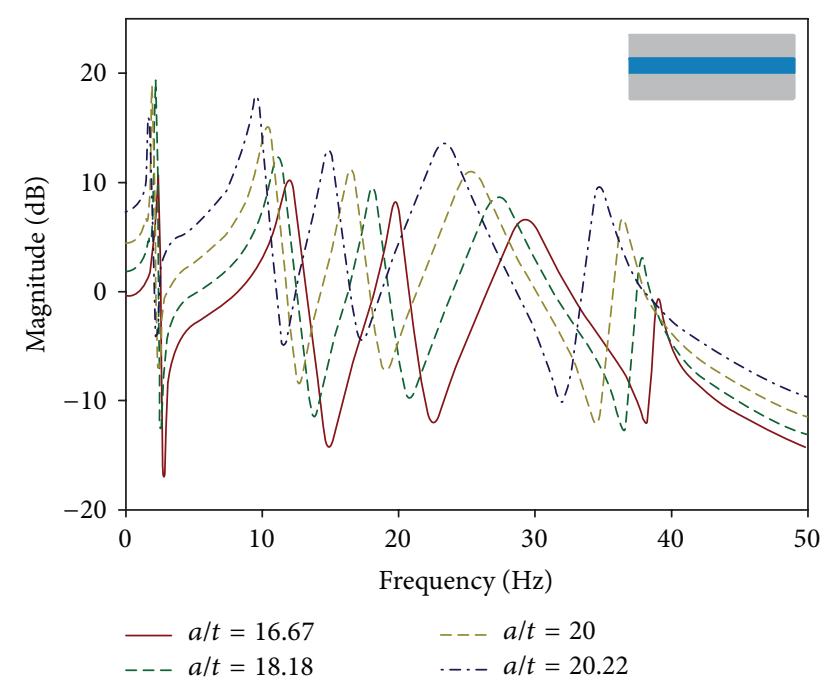

FIGURE 11: Frequency response of cylindrical aluminum panel in case of SSDL with variety of thickness ratios $a / t(a=0.2 \mathrm{~m}, \phi=$ $0.01 \mathrm{rad}, R=20 \mathrm{~m}, t_{2}=2 \mathrm{~mm}, t=t_{1}+t_{2}+t_{3}$, and $\left.\eta=0.1\right)$.

variety of the thicknesses ratio. With increasing of the thickness ratio, the natural frequency has decreasing trend, because the thickness of panel becomes thin. Figure 12 shows the frequency response of the cylindrical aluminum panel in case of SDDL with variety of the damping factors of the viscoelastic material. As the damping factor increases, the magnitude of each mode decreased. Figure 13 shows the comparison of the frequency response of the cylindrical aluminum panel in case of pure aluminum, SSDL, and SDDL. The results show that the sandwiched viscoelastic damping layer has good damping performance compared with no damped thick aluminum panel. Figure 14 shows the mode shapes in case of NDL, SSDL, and SDDL. The first, the second, and the fourth modes show the bending mode and the third and the fifth modes show a twisting mode.

\section{Conclusion}

In this paper, the vibration characteristics of a sandwiched cylindrical aluminum shell with viscoelastic damping treatment were investigated using layerwise theories. The transverse shear, the normal strains, and the curved geometry are exactly taken into account in the present layerwise shell model, which can depict the zig-zag in-plane and out-ofplane displacements. The frequency response functions, the mode shapes, and the modal loss factor of a cylindrical sandwich aluminum panel were investigated with viscoelastic damping treatment. The damped natural frequencies, the modal loss factors, and the frequency response functions of the cylindrical viscoelastic aluminum shells are compared with those of the base thick aluminum panel without a viscoelastic layer. The thickness and the damping ratio of the viscoelastic damping layer, the curvature of proposed cylindrical aluminum structure, and placement of damping

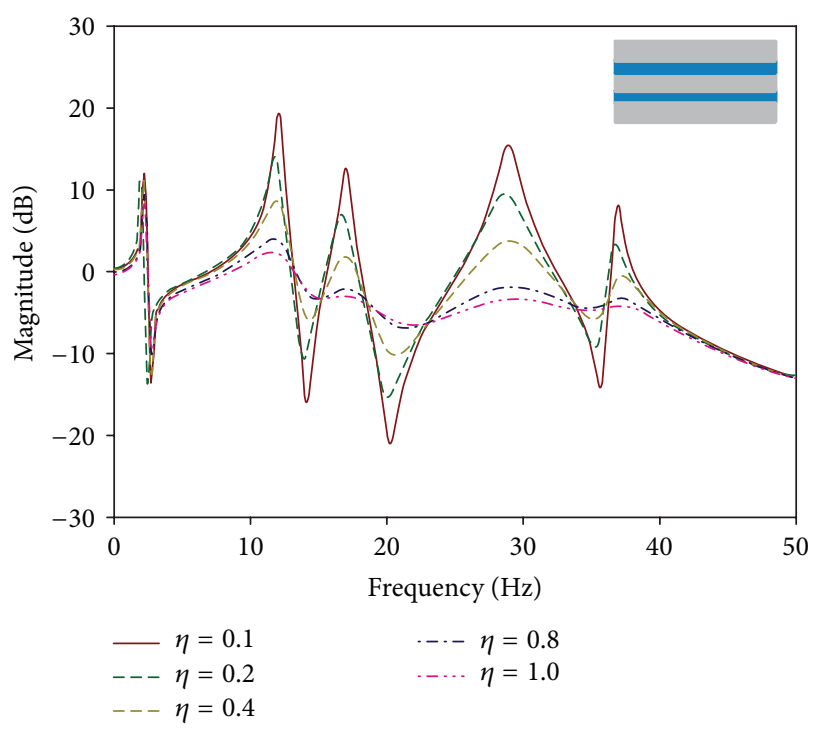

FIGURE 12: Frequency response of cylindrical aluminum panel in case of SDDL with variety of damping factors $\eta$ of viscoelastic material ( $a=0.2 \mathrm{~m}, \phi=0.01 \mathrm{rad}, R=20 \mathrm{~m}, t_{4}=3 \mathrm{~mm}, t_{5}=1 \mathrm{~mm}$, $t_{6}=4 \mathrm{~mm}, t_{7}=1 \mathrm{~mm}$, and $\left.t_{8}=3 \mathrm{~mm}\right)$.

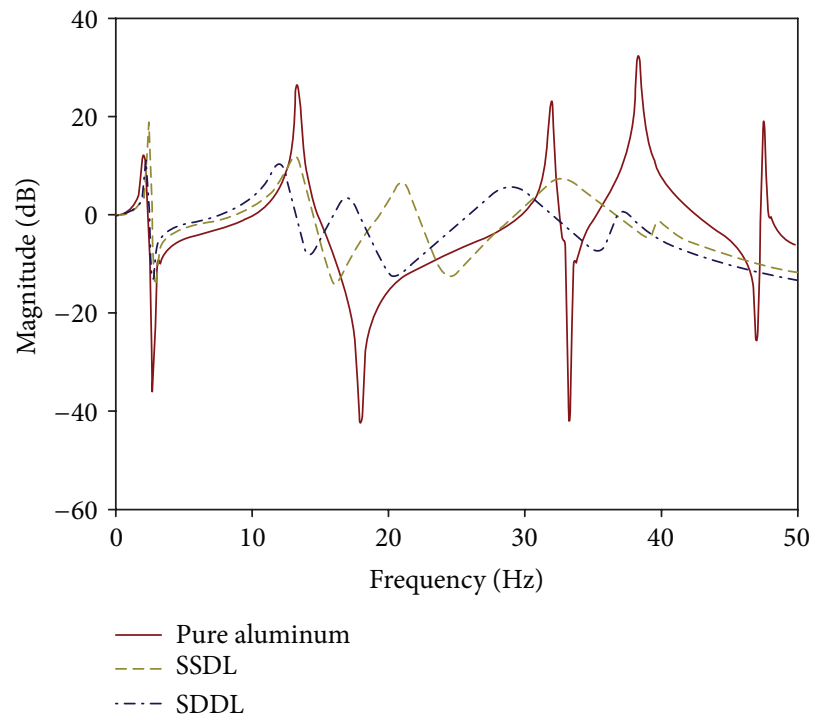

FIGURE 13: Frequency response of cylindrical aluminum panel in case of pure aluminum, SSDL, and SDDL $(a=0.2 \mathrm{~m}, \phi=0.01 \mathrm{rad}$, $R=20 \mathrm{~m}, t_{1}=5 \mathrm{~mm}, t_{2}=2 \mathrm{~mm}, t_{3}=5 \mathrm{~mm}, t_{4}=3 \mathrm{~mm}, t_{5}=1 \mathrm{~mm}$, $t_{6}=4 \mathrm{~mm}, t_{7}=1 \mathrm{~mm}$, and $\left.t_{8}=3 \mathrm{~mm}\right)$.

layer of the aluminum panel were investigated using frequency response function. The presented results show that the sandwiched viscoelastic damping layer can effectively suppress vibration of the cylindrical aluminum structure. 


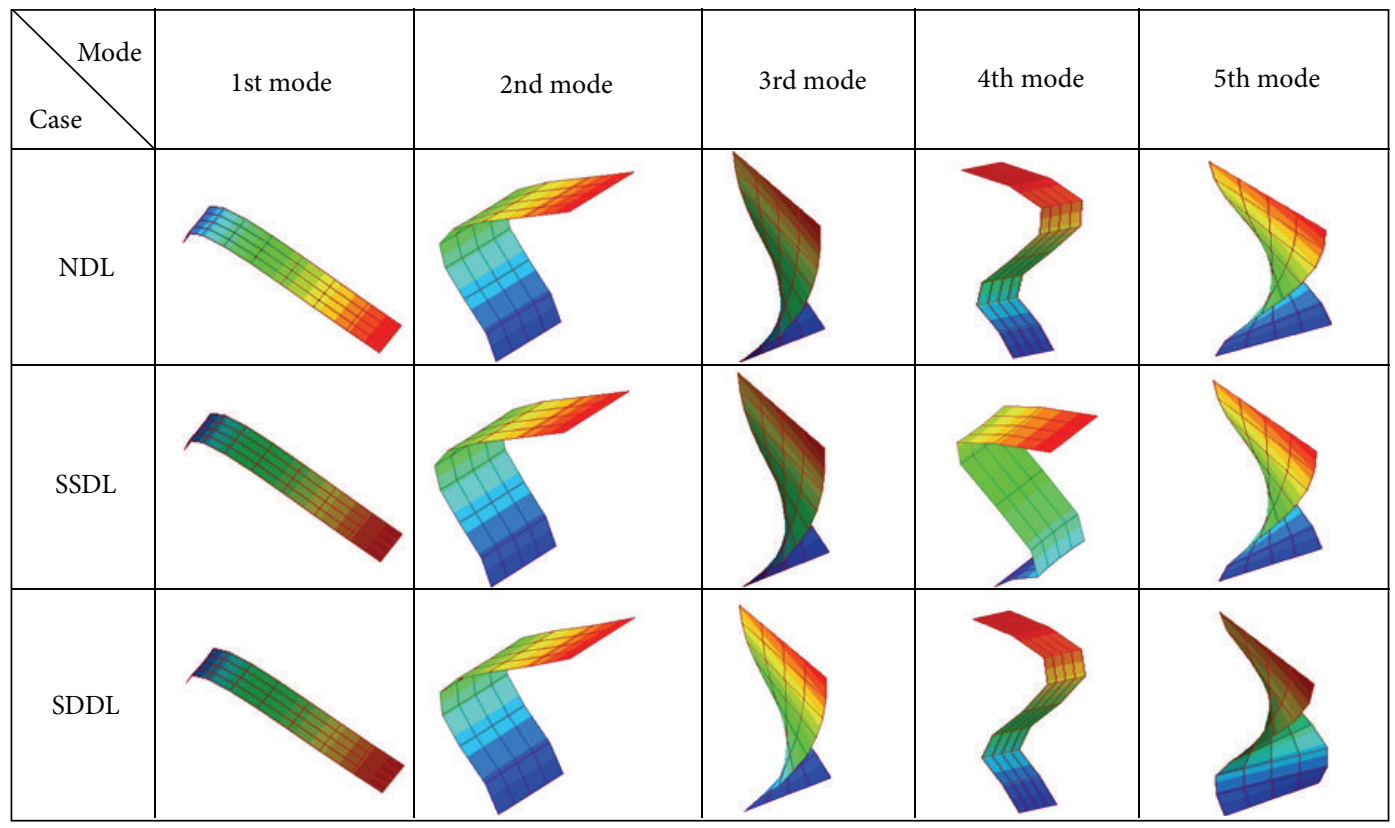

FIGURE 14: Mode shapes in case of NDL, SSDL, and SDDL.

\section{Acknowledgments}

This material is based on the works funded by Zhejiang Provincial Natural Science Foundation of China under Grant no. LQ12E05013, Research on Public Welfare Technology Application Projects of Zhejiang Province of China under Grant no. 2013C31081 and Wenzhou Planned Science and Technology Project of China under Grant no. H20110005.

\section{References}

[1] A. E. H. Love, "The small free vibrations and deformations of thins elastic shell," Philosophical Transactions of the Royal Society, vol. 179, pp. 491-546, 1888.

[2] R. N. Arnold and G. B. Warburton, "Flexural vibrations of the walls of thin cylindrical shells having freely supported ends," Proceedings of the Royal Society of London A, vol. 197, no. 1049, pp. 238-256, 1949.

[3] H. Chung, "Free vibration analysis of circular cylindrical shells," Journal of Sound and Vibration, vol. 74, no. 3, pp. 331-350, 1981.

[4] A. Bhimaraddi, "A higher order theory for free vibration analysis of circular cylindrical shells," International Journal of Solids and Structures, vol. 20, no. 7, pp. 623-630, 1984.

[5] K. P. Soldatos and V. P. Hadjigeorgiou, "Three-dimensional solution of the free vibration problem of homogeneous isotropic cylindrical shells and panels," Journal of Sound and Vibration, vol. 137, no. 3, pp. 369-384, 1990.

[6] R. Ross, E. E. Ungar E, and E. M. Kerwin, "Damping of plate of flexural vibrations by means of viscoelastic laminate," in Proceedings of the Structural Damping-A Colloquium on Structural Damping Held at the ASME Annual Meeting, pp. 4988, 1959.

[7] D. J. Mead and S. Markus, "Loss factors and resonant frequencies of encastré damped sandwich beams," Journal of Sound and Vibration, vol. 12, no. 1, pp. 99-112, 1970.
[8] W. Blasingame and R. A. DiTaranto, "Composite loss factors of selected laminated beams," The Journal of the Acoustical Society of America, vol. 36, no. 5, pp. 1052-1052, 1964.

[9] C. C. Siu and C. W. Bert, "Sinusoidal response of compositematerial plates with material damping," Journal of Engineering for Industry, vol. 96, no. 2, pp. 603-610, 1974.

[10] Y.-C. Chen and S.-C. Huang, "An optimal placement of CLD treatment for vibration suppression of plates," International Journal of Mechanical Sciences, vol. 44, no. 8, pp. 1801-1821, 2002.

[11] H. Zheng, C. Cai, G. S. H. Pau, and G. R. Liu, "Minimizing vibration response of cylindrical shells through layout optimization of passive constrained layer damping treatments," Journal of Sound and Vibration, vol. 279, no. 3-5, pp. 739-756, 2005.

[12] J. Ro and A. Baz, "Optimum placement and control of active constrained layer damping using modal strain energy approach," Journal of Vibration and Control, vol. 8, no. 6, pp. 861-876, 2002.

[13] R. S. Masti and M. G. Sainsbury, "Vibration damping of cylindrical shells partially coated with a constrained viscoelastic treatment having a standoff layer," Thin-Walled Structures, vol. 43, no. 9, pp. 1355-1379, 2005.

[14] S. Yin, T. T. Yu, and P. Liu, "Free vibration analyses of FGM thin plates by isogeometric analysis based on classicalplate theory and physical neutral surface," Advances in Mechanical Engineering, vol. 2013, Article ID 634584, 10 pages, 2013.

[15] J. N. Reddy, Mechanics of Laminated Composite Plates and Shells: Theory and Analysis, CRC Press, Boca Raton, Fla, USA, 2004. 

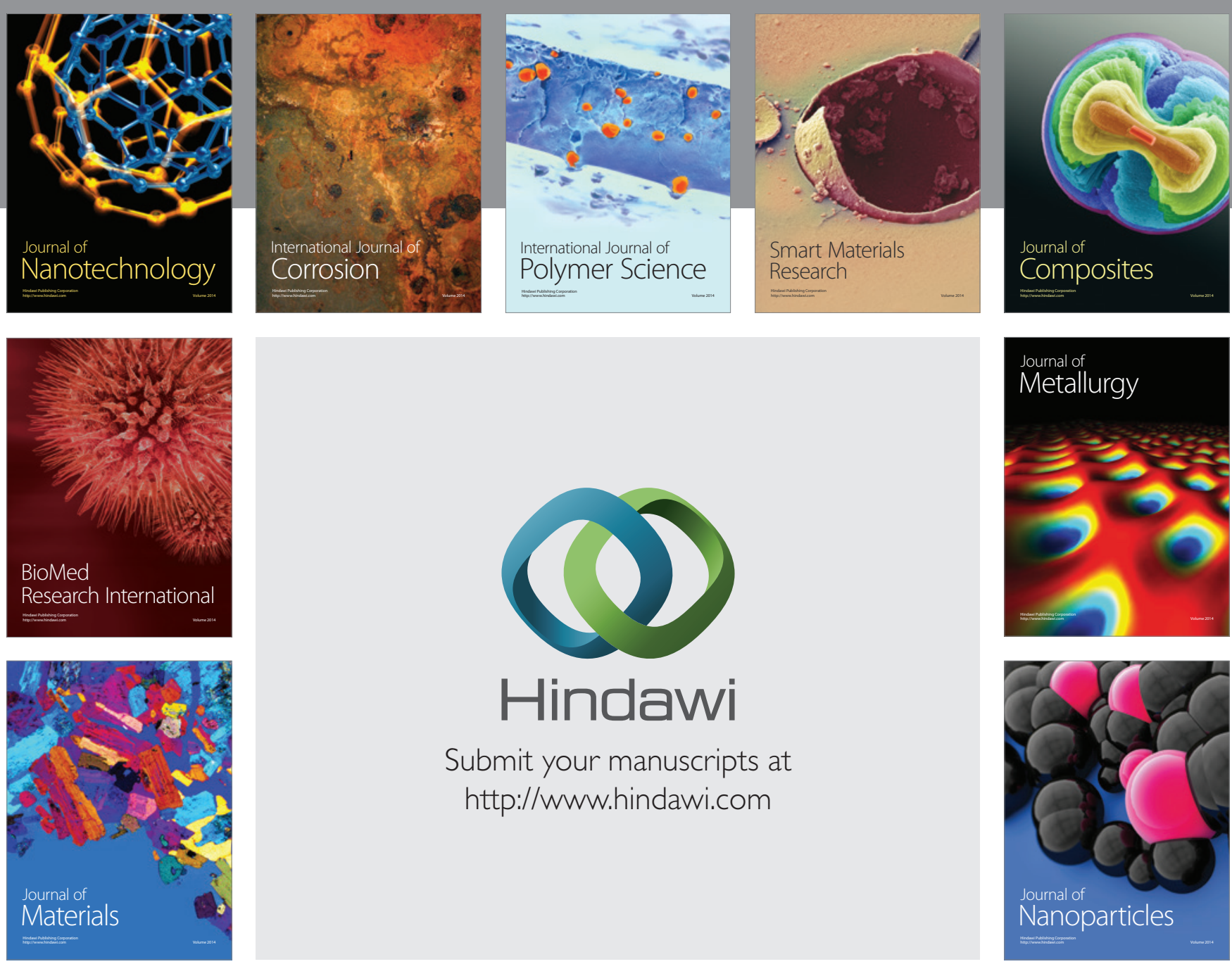

Submit your manuscripts at http://www.hindawi.com
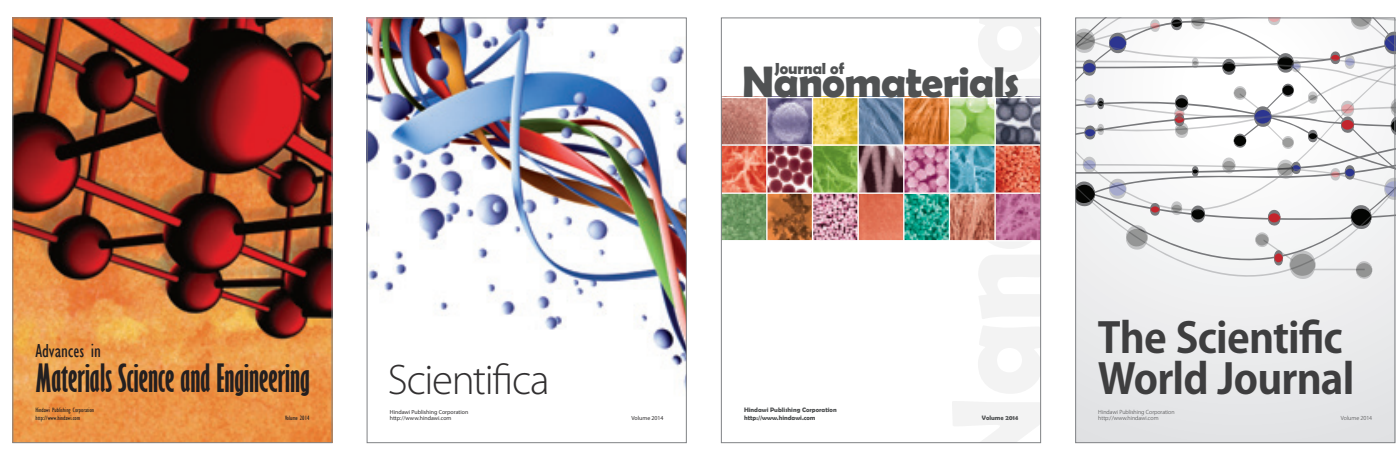

\section{The Scientific World Journal}
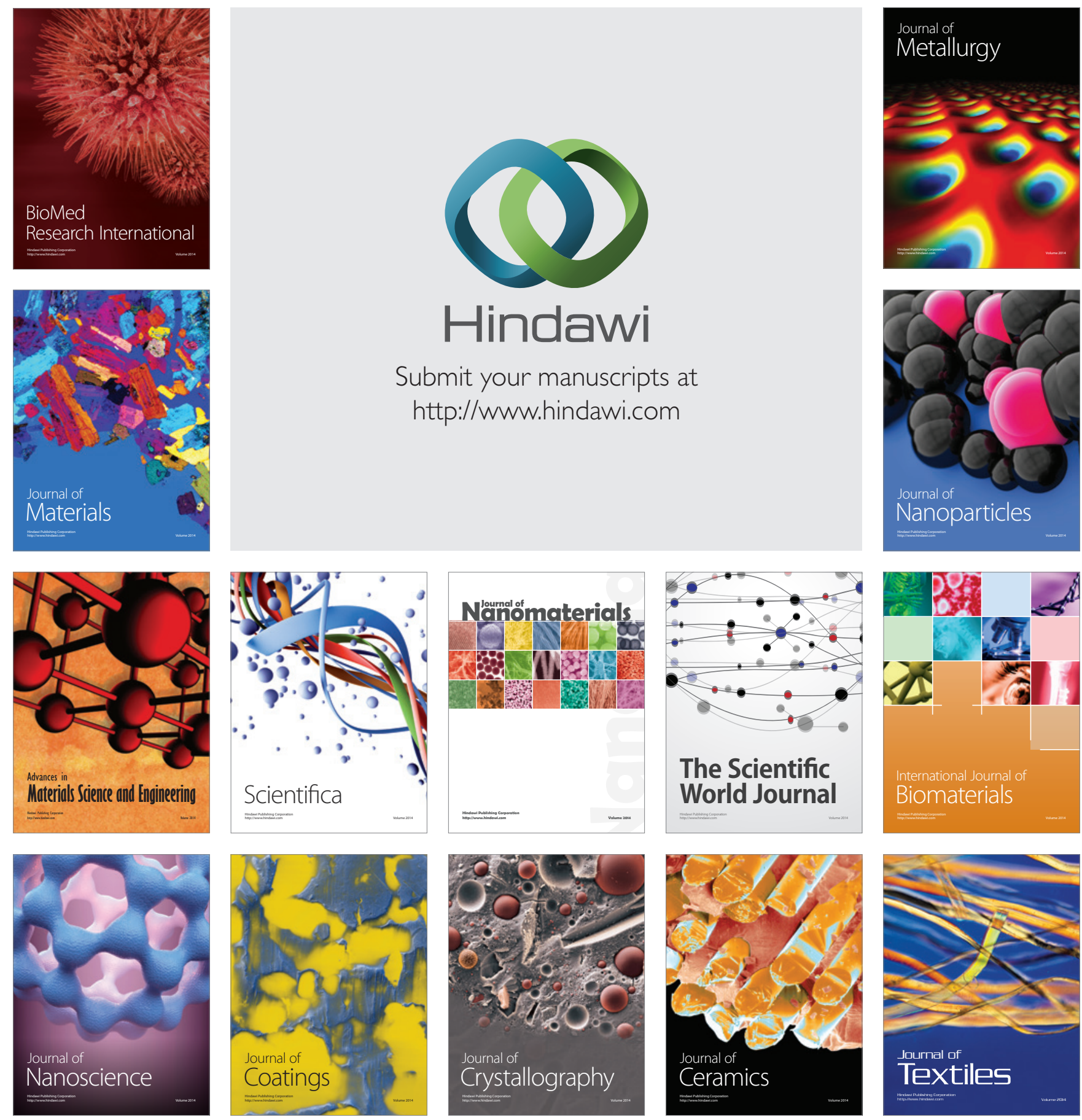